\title{
5D-STEM: Live processing and display at 15,000 diffraction patterns per second
}

Andreas Mittelberger ${ }^{1}$, Benedikt Haas ${ }^{2}$, Benjamin Plotkin-Swing ${ }^{1}$, Chris Meyer ${ }^{3}$, Niklas Dellby ${ }^{1}$, Luca Piazza $^{4}$, Ondrej Krivanek ${ }^{1}$, Christoph Koch ${ }^{2}$ and Tracy Lovejoy ${ }^{1}$

${ }^{1}$ Nion R\&D, 11511 NE 118th St, Kirkland, WA, 98034, USA, United States, ${ }^{2}$ Department of Physics, Humboldt University of Berlin, Berlin, Germany, United States, ${ }^{3}$ Nion R\&D, 11511 NE 118th St, Kirkland, WA, 98034, USA, Washington, United States, ${ }^{4}$ DECTRIS Ltd., Taefernweg 1, 5405 Baden-Daettwil, Switzerland, United States

Compared to traditional Scanning Transmission Electron Microscopy (STEM) with a handful of fixed-shape detectors, acquiring whole diffraction patterns on a 2-dimensional (2D) detector enables advantageous imaging methods such as ptychography, center-of-mass (COM) analysis, and other 4D-STEM techniques.

When performed sufficiently fast, the time dependence of 4D-STEM can be studied and the technique is then best called 5D-STEM. The ideal 5D-STEM system should have the following properties:

- Comparable speed to traditional STEM detectors, 100,000 frames/s, with little or no read noise.

- Detection quantum efficiency close to 1.

- Sufficient dynamic range to efficiently record bright-field and dark-field signals simultaneously.

- Enough pixels to acquire detailed diffraction patterns, 100x100 or more.

- The pattern should be energy filtered for the elastic peak to reduce the inelastic background fuzz.

- The system should deliver the processed results to the user live for immediate quality assessment, and efficiently compress the data for subsequent analysis whenever possible.

We report on a system that approaches all these goals. The system uses a Nion IRIS spectrometer for selecting the elastic signal, the Nion Swift open-source software for processing and display, and a DECTRIS ELA detector. The detector has 1,028x512 pixels, $107: 1$ dynamic range, and operates in event counting mode up to $\sim 0.8 \mathrm{pA} / \mathrm{pixel}$ with virtually zero dark current or read noise [1]. Full frames can be readout at 2,250 frames per second (FPS) with 16-bit numbers $(\sim 2.2 \mathrm{~GB} / \mathrm{s})$, and the speed increases linearly with decreased readout size (in the short dimension) and bit depth up to 18,000 fps for a readout 1,028x130 and 8-bit depth. In 8-bit mode, running with a bright field disk spread over a 20 pixel radius (1,200 pixels total), a beam current up to 500 pA can be accommodated.

Figure 1 shows an example 5D dataset mapping the electric field and charge density in twisted bilayer graphene, acquired using a centered 130x130 detector readout, 15,000 patterns/s, 8-bit data, and 512x512 scan pixels (i.e. $17 \mathrm{~s} / \mathrm{scan}$ ). Fifteen such frames were acquired (total time $\sim 4.5$ minutes), aligned and summed to improve the signal to noise. Time resolution on the scale of $1 \mathrm{~s}$ is useful for dealing with drift during in-situ experiments. As described below, the processed output was available live, which is invaluable for determining if the resulting dataset is good (or not). The full 5D dataset would have been $\sim 250 \mathrm{~GB}$.

At this data rate, typical hard drives will fill up in minutes - data storage and processing can quickly become intractable, especially for a new user. We have developed a user-friendly interface for pre-defining common processing operations, which allows live processing and display as the data is being acquired, and can reduce the size of the resulting datasets by a factor of $\sim 104$ or more. It works as follows.

Event-counted frames from the detector are streamed to a dedicated server, compressed, and streamed to the user PC via a 10 Gbit Ethernet connection. On the user PC the data is decompressed and processed. All processing is done on a standard workstation PC using the Python-based open source software Nion Swift [2]. Primary preprocessing like hot-pixel interpolation and gain correction are done automatically, and the full (compressed) 4D dataset is streamed to the hard-drive in case it is needed later. The user has the option to, also or instead, request reduced data. Figure 1 shows two common reduced data options: virtual Medium Angle Annular Dark Field detector (vMAADF, $45 \mathrm{mrad}<\beta / 2<80 \mathrm{mrad}$ ), and center of mass $(\mathrm{COM})$ of the bright-field disk. These three images, vMAADF, $\operatorname{COM}(\mathrm{X})$, and $\operatorname{COM}(\mathrm{Y})$ are all that is needed for alignment (rigid registration) and for generating the electric field and charge density maps in Figure 1, while providing immediate data savings of $\sim 6,000 x$ (i.e. three 
data channels instead of 130x130). Additional processing, such as non-rigid registration, can be applied using these three data channels, and the live data reduction allows for longer acquisition times compared to full 4D data. [3]

Speedy 5D acquisition with the latest generation of detectors, together with live processing has the potential to make 5D-STEM an everyday technique. Time series are a common acquisition mode, and other parameters can also be varied between 4D-STEM scans such as scan rotation, beam tilt (i.e. precession), etc., for other types of 5D-STEM. A variety of examples will be shown at the meeting.
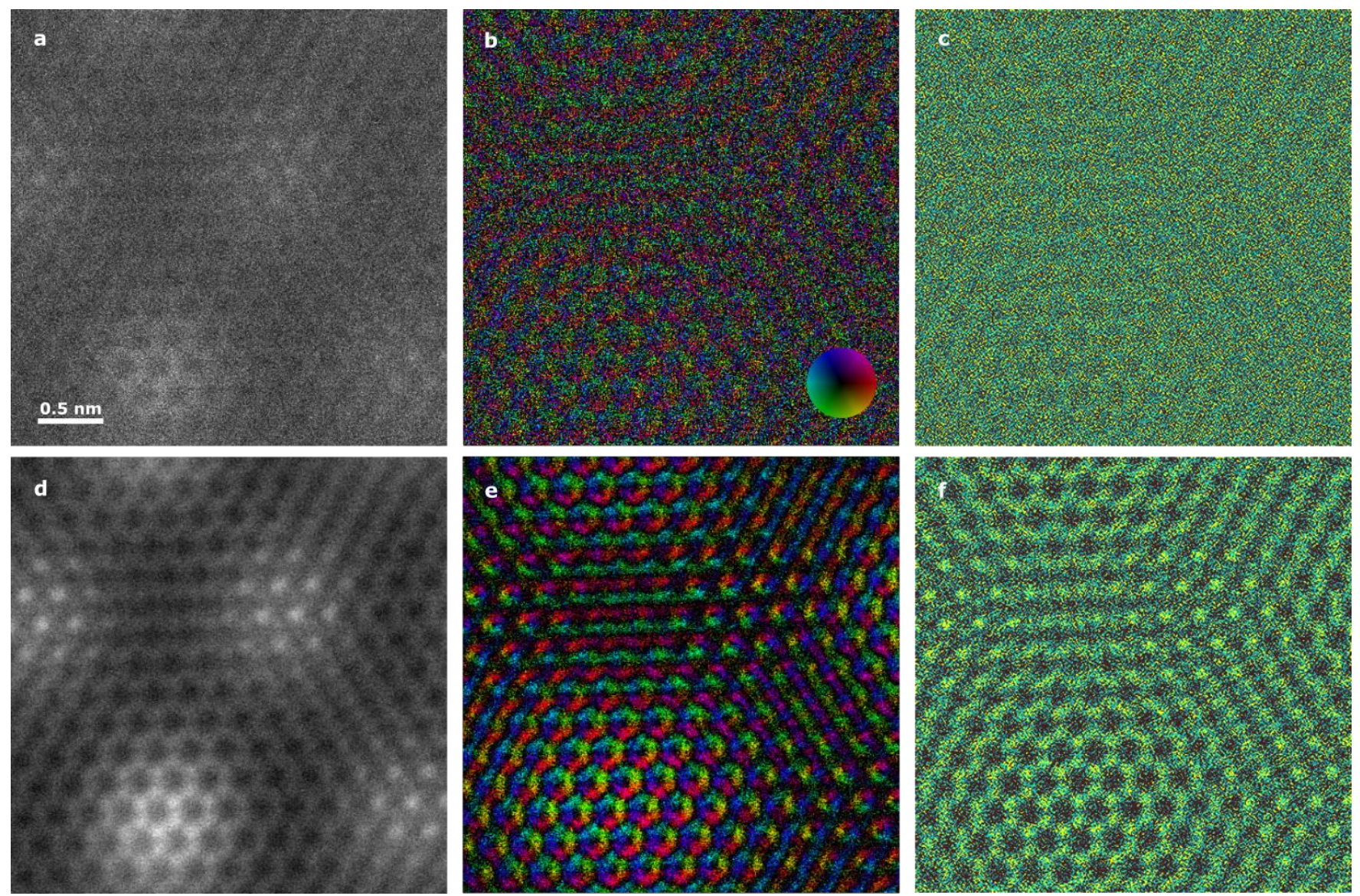

Figure 1. Figure 1. Live-Processed 5D-STEM data from twisted bilayer graphene ( 8.8 degree rotation). The data was acquired at 15,000 frames/s, and the center-of-mass and virtual MAADF filters applied live for a 6,000x reduction in the size of the dataset. Top row shows data calculated from a single 4D-STEM frame: a) virtual MAADF image, $b$ ) beam deflection (proportional to electric field) and c) charge density. Bottom row shows data calculated from 15 aligned (rigid registration) and summed 4D STEM frames: d) virtual MAADF, e) beam deflection (proportional to electric field) and f) charge density. $E 0=60 \mathrm{kV}, \mathrm{Ip}=20 \mathrm{pA}, \alpha / 2=36 \mathrm{mrad}$.

\section{References}

[1] B. Plotkin-Swing et al., Ultramicroscopy 217, 113067 (2020).

[2] C. Meyer et al., Microscop. Microanal. 25 (Suppl 2), 122 (2019). https://github.com/nion-software/nionswift

[3] B. Haas et al., Microscop. Microanal. 26 (Suppl 2), 1898 (2020), and this meeting. 